\title{
The added value of pirfenidone to fight inflammation and fibrotic state induced by SARS-CoV-2
}

\section{Anti-inflammatory and anti-fibrotic therapy could solve the lung complications of the infection?}

\author{
Francesco Ferrara $^{1}$ (D) $\cdot$ Giovanni Granata $^{2} \cdot$ Chiara Pelliccia $^{3} \cdot$ Raffaele La Porta $^{4} \cdot$ Antonio Vitiello $^{1}$
}

Received: 1 May 2020 / Accepted: 23 June 2020 / Published online: 27 June 2020

(C) Springer-Verlag GmbH Germany, part of Springer Nature 2020

\begin{abstract}
Aim SARS-CoV-2 infection has been divided by scientific opinion into three phases: the first as asymptomatic or slightly symptomatic and the second and the third with greater severity, characterized by a hyperinflammatory and fibrotic state, responsible for lung lesions, in some cases fatal. The development of antiviral drugs directed against SARS-CoV-2 and effective vaccines is progressing; meanwhile, the best pharmacological objective is related to the management of all the complications caused by this viral infection, mainly controlling the inflammatory and fibrotic state and preventing the infection from moving into the most serious phases.

Subject and method Describe the scientific rationale related to the use of an antifibrotic therapy with pirfenidone, as monotherapy and/or in combination with anti-inflammatory drugs to manage and control complications of SARS-CoV-2 infection.

Results Based on the scientific literature and epidemiological results and considering the pathophysiological, biological, and molecular characteristics of SARS-CoV-2, an antifibrotic drug such as pirfenidone as monotherapy or in combination with antiinflammatory drugs can be (acting early, at the right doses and at the right time) therapeutically effective to avoid serious complications during viral infection. The same approach can also be effective as postinfection therapy in patients with residual pulmonary fibrotic damage. Management of inflammation and fibrotic status with a combination therapy of pirfenidone and IL-6 or IL-1 inhibitors could represent a pharmacological synergy with added value.

Conclusion In this article, we consider the role of antifibrotic therapy with pirfenidone in patients with SARS-CoV-2 infection on going or in the stage of postinfection with pulmonary fibrotic consequences. The scientific rationale for its use is also described.
\end{abstract}

Keywords Pirfenidone $\cdot$ Fibrotic $\cdot$ Inflammation $\cdot$ Cytokine $\cdot$ Interleukin $\cdot$ SARS-CoV-2

\section{Introduction}

The current global SARS-CoV-2 pandemic (COVID-19) represents a health crisis with few precedents in history;

A preliminary version of this report was made publicly available as a socalled preprint at a nonprofit repository (Authorea) with DOI number: https://doi.org/10.22541/au.158802198.88770371

Francesco Ferrara

francesco.ferrara@uslumbria1.it

1 Usl Umbria 1, Perugia, Italy

2 Asl Salerno, Salerno, Italy

3 Usl Umbria 2, Terni, Italy

4 Asur Marche, Ancona, Italy at the time of writing this article, SARS-CoV-2 caused about 6.42 million infected and about 383,000 deaths [1]. SARS-CoV-2 infection, in the most severe cases, can cause tissue hyperinflammation, fibrosis and scarring, lung collapse, multi-organ dysfunction, and patient death. [2] To date, no effective or antiviral vaccines against SARS-CoV-2 are available, so it is very important to decrease the aggressiveness of the viral infection, avoiding serious complications and patient death. The excessive inflammatory state, associated with the presence of fibrotic tissue induced by SARS-CoV-2, has been shown to play a key role in clinical cases considered more critical. [3] In the more severe stages of viral infection, excessive release of pro-inflammatory mediators, such as cytokines, leads to lung damage with extensive fibrosis and rapid onset of respiratory distress syndrome. [4, 5] The use of agents to prevent or reduce fibrotic status, such 
as pirfenidone, may be therapeutically effective in preventing serious or fatal complications. Pirfenidone is the drug of choice in the treatment of idiopathic pulmonary fibrosis (IPF) [6] and with a pleiotropic mechanism of action reduces the fibrotic and inflammatory state of lung tissue. Its therapeutic efficacy could also benefit healed patients with consequences on lung tissue with a state of fibrosis. In addition, a combination therapy of anti-inflammatories and antifibrotics such as pirfenidone could lead to additional clinical benefits, using low dosages and decreasing the risk of toxicity.

\section{The clinical aspects of the three phases of SARS-CoV-2 infection and similarities with IPF}

The first cases of SARS-CoV-2 infection were recorded in November 2019 in China; then, rapidly, they spread to all countries around the world causing thousands of deaths. SARS-CoV-2 infection in the most severe stages can rapidly cause respiratory distress syndrome. According to observational studies conducted, the majority of patients considered severe cases present bilateral interstitial pneumonia and a hyperactive inflammatory state that is not only localized in lung tissue but in all tissues of the body causing multi-organ dysfunction [7, 8]. SARS-CoV-2 infection has, according to scientific advice, been divided into three stages, the first as asymptomatic or slightly symptomatic with symptoms such as mild sore throat and abdominal pain and the second and third more severe stages presenting a generalized inflammatory state and respiratory distress syndrome. Studies have shown that bilateral interstitial pneumonia, so called because it attacks the tissue covering the pulmonary alveoli, is associated with the presence of fibrotic tissue caused by excess collagen (fibrosis) in the pulmonary interstitium with hyperinflammation present [9]. Phases two and three are the most severe, and the presence of inflammation in the lungs and fibrotic tissue requires timely immunomodulatory and anti-inflammatory treatment. However, based on descriptions of symptomatology and diagnostic investigations of early observational studies, one might think that the use of a drug currently indicated for pulmonary fibrosis such as pirfenidone could bring great additional benefits both during viral infection and in the post-healing phase with residual pulmonary fibrotic damage [10]. The rationale for using antifibrotic therapy is based on the characteristics of pulmonary fibrosis observed in COVID-19, ranging from fibrosis associated with organic pneumonia to severe acute lung injury, where a rapid evolution towards widespread fibrotic change is observed [11]. In the most severe and fatal cases, pulmonary fibrosis is generally present [12] and also severe fibrotic organic pneumonia. The presence of pulmonary fibrosis is probably a consequence of the cytokine storm. Some of these biological and pathological features are shared with idiopathic pulmonary fibrosis (IPF) such as chronic inflammatory fibrotic lung disease caused by the synthesis and release of proinflammatory cytokines, including tumor necrosis factor alpha $(\mathrm{TNF}-\alpha)$ and interleukin-1-beta (IL-1 $\beta)$. Antifibrotic therapy with pirfenidone, a drug indicated for the treatment of IPF, could therefore play a key role in preventing serious or fatal lung complications. However, antifibrotic therapy could play an even more important role in combined regimens, once identified, with effective anti-inflammatory treatments. Combination therapy could act on the main antiinflammatory and antifibrotic pathways in a synergistic way, mitigating the consequences of pulmonary fibrosis.

\section{The potential therapeutic role of pirfenidone to fight SARS-CoV-19}

Pirfenidone, for the treatment of IPF, is administered orally, 3 times a day, 2 or 3 tablets each time, for a minimum of 4 weeks. The mechanism of action of pirfenidone has not yet been fully determined and clarified. However, existing data indicate that pirfenidone has both antifibrotic and antiinflammatory properties, which have been tested in a variety of in vitro systems and animal models of pulmonary fibrosis (bleomycin-induced fibrosis and transplant fibrosis). Pirfenidone has been shown to reduce the accumulation of inflammatory cells in response to various stimuli [13]. Pirfenidone is able to mitigate the proliferation of fibroblasts, the production of proteins, and cytokines associated with fibrosis; it also mitigates the increase in biosynthesis and the accumulation of extracellular matrix in response to cytokine growth factors such as transformative growth factor beta (TGF- $\beta$ ) and platelet-derived growth factor (PDGF) [14]. There are currently no standardized therapeutic protocols to manage phases two and three of COVID-19 infection. Several clinical trials are underway to define the most appropriate drugs and doses to be used in the different stages of infection. However, it is certain that COVID-19 lung lesions are caused by a cytokinic storm leading to a hyperinflammatory and fibrotic state; therefore, control of this phase is essential to avoid serious complications and patient death.

To date, the therapeutic efficacy of pirfenidone in pulmonary fibrosis induced by SARS-CoV-2 is still being demonstrated in clinical trials. Nevertheless, some evidence has already demonstrated the efficacy of antifibrotics in patients with pathogenic profibrotic pathways caused by immune/ inflammatory dysregulation, which may have similarities with those caused by SARS-CoV-2 infection [15, 16]. In addition, available studies potentially suggest that antifibrotic therapy, if used at the onset of SARS-CoV-2 infection, could be very 
Fig. 1 Pleiotropic effects of pirfenidone

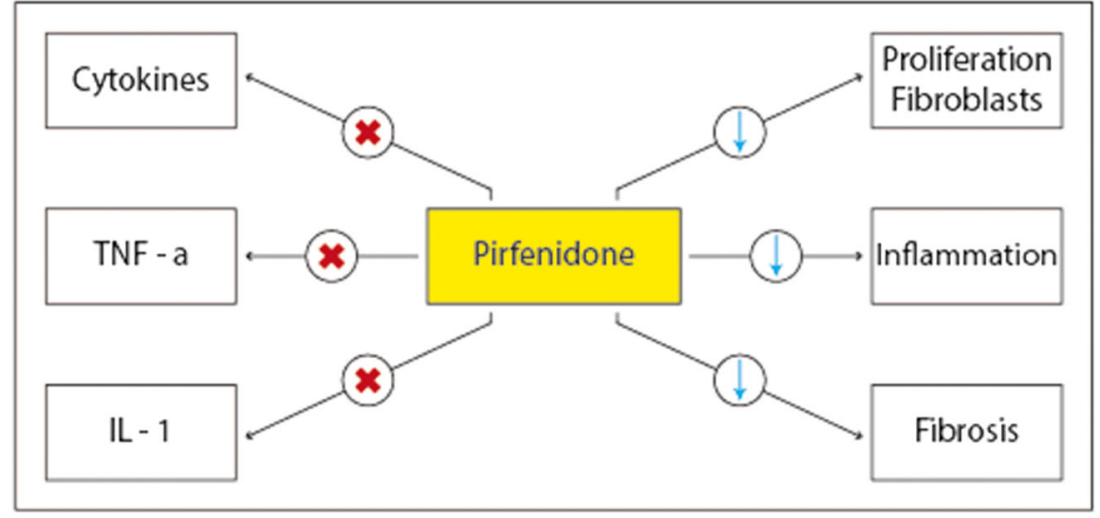

effective in reducing and preventing fibrotic damage caused by inflammatory/immune dysregulation [17, 18]. A key aspect to consider is the timing of the onset of the antifibrotic action and the therapeutic efficacy of pirfenidone. In some severe cases of SARS-CoV-2, the evolution of the infection may be very rapid, and pirfenidone may not act with rapid efficacy. Again, evidence shows that pirfenidone has pleiotropic effects [19] in reducing inflammation and antioxidant action associated with a reduction in the fibrotic state (Fig. 1).

These pleiotropic effects could add value to the therapeutic potential against COVID-19 [13]. In addition, immunomodulants such as IL- 1 and IL-6 inhibitors, or antiinflammatory, could be combined with pirfenidone to have an impact therapy that acts early on inflammation and fibrotic state. Finally, studies have shown that some patients cured of COVID-19 had pulmonary fibrotic tissue residues; also in this category of patients, the use of pirfenidone as therapeutic treatment after infection could be effective. For a possible relevance to the pulmonary outcomes of COVID-19, it should be noted that, recently, for pirfenidone, the revolutionary therapy status (which accelerates the drug approval process) for fibrous interstitial lung disease has been recognized by the FDA. However, some issues remain to be clarified. First, what is the effect of antifibrotic molecules such as pirfenidone on internalization and viral replication COVID-19?

\section{Conclusions}

The global COVID-19 pandemic is a health and economic emergency. Many features of biological processes leading to acute respiratory distress syndrome caused by SARS-CoV-2 infection are partly shared with IPF. Antifibrotic drugs such as pirfenidone may have therapeutic potential to prevent or reduce fibrotic lung lesions of the ongoing COVID-19 infection, or in patients already healed but with fibrotic consequences. In conclusion, we hope that the observations highlighted will induce the scientific world to work on well-designed studies of antifibrotic therapies for severe COVID-19 pneumonia.
Authors' contributions I, the undersigned, Francesco Ferrara, and any other author declare that the manuscript was written entirely by the authors. All authors made an equal contribution in the development of the paper.

Compliance with ethical standards The document does not conflict with ethical legislation.

Conflict of interest The authors declare that they have no conflict of interest.

Ethical approval There are no sensitive data, and no patients were recruited for this study. The authors accept the full TRANSFER OF COPYRIGHT to the journal.

\section{References}

1. World Health Organization HO (2020) Coronavirus disease 2019 (COVID-19) situation report Available from https://www.who.int/ emergencies/diseases/novel-coronavirus-2019/situation-reports

2. Li YC, Bai WZ, Hashikawa T (2020) The neuroinvasive potential of SARS-CoV2 may play a role in the respiratory failure of COVID-19 patients. J Med Virol

3. Zhu N, Zhang D, Wang W, Li X, Yang B, Song J, Zhao X, Huang B, Shi W, Lu R, Niu P, Zhan F, Ma X, Wang D, Xu W, Wu G, Gao GF, Tan W, China Novel Coronavirus Investigating and Research Team (2020) A novel coronavirus from patients with pneumonia in China, 2019. N Engl J Med 382:727-733

4. Yang X, Yu Y, Xu J et al (2020) Clinical course and outcomes of critically ill patients with SARS-CoV-2 pneumonia in Wuhan, China: a single centered, retrospective, observational study. Lancet Respir Med 8:457-481

5. Livingston E, Bucher K (2020) Coronavirus disease 2019 (COVID19) in Italy. JAMA 323:1335

6. Chung MP, Park MS et al (2020) Safety and efficacy of pirfenidone in advanced idiopathic pulmonary fibrosis: a nationwide postmarketing surveillance study in Korean patients. Adv Ther 15

7. Wang D, Hu B et al (2020) Clinical characteristics of 138 hospitalized patients with 2019 novel coronavirus-infected pneumonia in Wuhan, China. JAMA

8. Chen N, Zhou M, Dong X, Qu J, Gong F, Han Y, Qiu Y, Wang J, Liu Y, Wei Y, Xia J', Yu T, Zhang X, Zhang L (2020) Epidemiological and clinical characteristics of 99 cases of 2019 novel coronavirus pneumonia in Wuhan, China: a descriptive study. Lancet. 395(10223):507-513 
9. Guan WJ, Ni ZY, Hu Y, Liang WH, Ou CQ, He JX, Liu L, Shan H, Lei CL, Hui DSC, du B, Li LJ, Zeng G, Yuen KY, Chen RC, Tang CL, Wang T, Chen PY, Xiang J, Li SY, Wang JL, Liang ZJ, Peng YX, Wei L, Liu Y, Hu YH, Peng P, Wang JM, Liu JY, Chen Z, Li G, Zheng ZJ, Qiu SQ, Luo J, Ye CJ, Zhu SY, Zhong NS, China Medical Treatment Expert Group for Covid-19 (2020) Clinical characteristics of coronavirus disease 2019 in China. N Engl J Med 382:1708-1720

10. King CS, Nathan SD (2017) Idiopathic pulmonary fibrosis: effects and optimal management of comorbidities. Lancet Respir Med 5: 72-84

11. Shi H, Han X, Jiang N, Cao Y, Alwalid O, Gu J, Fan Y, Zheng C (2020) Radiological findings from 81 patients with COVID-19 pneumonia in Wuhan, China: a descriptive study. Lancet Infect Dis 20:425-434

12. Zhang T, Sun LX, Feng RE (2020) Comparison of clinical and pathological features between severe acute respiratory syndrome and coronavirus disease 2019. Chinese Journal of Tuberculosis and Respiratory Diseases

13. George PM, Wells AU (2017) Pirfenidone for the treatment of idiopathic pulmonary fibrosis. Expert Rev Clin Pharmacol 10(5): 483-491

14. Lancaster LH, de Andrade JA et al (2017) Pirfenidone safety and adverse event management in idiopathic pulmonary fibrosis. Eur Respir Rev 26(146)

15. Wells AU, Flaherty KR, Brown KK, Inoue Y, Devaraj A, Richeldi L, Moua T, Crestani B, Wuyts WA, Stowasser S, Quaresma M, Goeldner RG, Schlenker-Herceg R, Kolb M, Abe S, Aburto M, Acosta O, Andrews C, Antin-Ozerkis D, Arce G, Arias M, Avdeev S, Barczyk A, Bascom R, Bazdyrev E, Beirne P, Belloli E, Bergna MA, Bergot E, Bhatt N, Blaas S, Bondue B, Bonella F, Britt E, Buch K, Burk J, Cai H, Cantin A, Castillo Villegas DM, Cazaux A, Cerri S, Chaaban S, Chaudhuri N, Cottin V, Crestani B, Criner G, Dahlqvist C, Danoff S, Dematte D'Amico J, Dilling D, Elias P, Ettinger N, Falk J, Fernández Pérez ER, Gamez-Dubuis A, Giessel G, Gifford A, Glassberg M, Glazer C, Golden J, Gómez Carrera L, Guiot J, Hallowell R, Hayashi H, Hetzel J, Hirani N, Homik L, Hope-Gill B, Hotchkin D, Ichikado K, Ilkovich M, Inoue Y, Izumi S, Jassem E, Jones L, Jouneau S, Kaner R, Kang J, Kawamura T, Kessler R, Kim Y, Kishi K, Kitamura H, Kolb M,
Kondoh Y, Kono C, Koschel D, Kreuter M, Kulkarni T, Kus J, Lebargy F, León Jiménez A, Luo Q, Mageto Y, Maher TM, Makino S, Marchand-Adam S, Marquette C, Martinez R, Martínez M, Maturana Rozas R, Miyazaki Y, Moiseev S, MolinaMolina M, Morrison L, Morrow L, Moua T, Nambiar A, Nishioka Y, Nunes H, Okamoto M, Oldham J, Otaola M, Padilla M, Park JS, Patel N, Pesci A, Piotrowski W, Pitts L, Poonyagariyagorn H, Prasse A, Quadrelli S, Randerath W, Refini R, Reynaud-Gaubert M, Riviere F, Rodríguez Portal JA, Rosas I, Rossman M, Safdar Z, Saito T, Sakamoto N, Salinas Fénero M, Sauleda J, Schmidt S, Scholand MB, Schwartz M, Shapera S, Shlobin O, Sigal B, Silva Orellana A, Skowasch D, Song JW, Stieglitz S, Stone H, Strek M, Suda T, Sugiura H, Takahashi H, Takaya H, Takeuchi T, Thavarajah K, Tolle L, Tomassetti S, Tomii K, Valenzuela C, Vancheri C, Varone F, Veeraraghavan S, Villar A, Weigt S, Wemeau L, Wuyts W, Xu Z, Yakusevich V, Yamada Y, Yamauchi H, Ziora D (2020) Nintedanib in patients with progressive fibrosing interstitial lung diseases - subgroup analyses by interstitial lung disease diagnosis in the INBUILD trial: a randomised, double-blind, placebo-controlled, parallel-group trial. Lancet Respir Med 8:453-460

16. Maher TM, Corte TJ, Fischer A et al (2019) Pirfenidone in patients with unclassifiable progressive fibrosing interstitial lung disease: a double-blind, randomised, placebo-controlled, phase 2 trial. Lancet Respir Med 8:147-157

17. Lota HK et al (2013) The Evolving Pharmacotherapy of Pulmonary Fibrosis Review. Expert Opin Pharmacother 14(1):79-89

18. Nathan SD, Albera C, Bradford WZ, Costabel U, Glaspole I, Glassberg MK, Kardatzke DR, Daigl M, Kirchgaessler KU, Lancaster LH, Lederer DJ, Pereira CA, Swigris JJ, Valeyre D, Noble PW (2017) Effect of pirfenidone on mortality: pooled analyses and meta-analyses of clinical trials in idiopathic pulmonary fibrosis. Lancet Respir Med 5:33-41

19. Veit $\mathrm{T}$ et al (2019) Pirfenidone exerts beneficial effects in patients with IPF undergoing single lung transplantation. Am J Transplant

Publisher's note Springer Nature remains neutral with regard to jurisdictional claims in published maps and institutional affiliations. 